Bull. Mater. Sci., Vol. 8, No. 1, February 1986, pp. 49-52. (C) Printed in India.

\title{
Tunnelling of electrons from deep traps of $\mathrm{MgO}$
}

\author{
M N BAPAT and S SIVARAMAN \\ Department of Physics, Dr Harisingh Gour Vishwavidyalaya, Sagar 470003, India \\ MS received 11 September 1985

\begin{abstract}
A new result on electron tunnelling from the deep traps of $\mathrm{MgO}$, reported recently is discussed. The electrons from the $540 \mathrm{~K}$ trapping group are released spontaneously at $480 \mathrm{~K}$. The proposed mechanism for electron tunnelling is discussed in the light of existing theories. The location of the peak in the isothermal decay is found to depend on (i) the extent of "population of the source trap group; (ii) the availability of shallow trapping levels to the rushing electrons and (iii) the nature of traps.

The glow curve experiment indicates that the isothermal decay after saturation excitation causes reduction in the thermoluminescence intensity of the $540 \mathrm{~K}$ peak.
\end{abstract}

Keywords. Electron tunnelling; isothermal decay; thermoluminescence; trapping groups.

Phosphorescence has been used for studying the shallow traps in the crystal. The emission that persists after cessation of excitation is known as phosphorescence. The rate of decay of emission intensity depends on a variety of factors viz host composition, energy of excitation, concentration of traps, level of population, ambient temperature etc. The rate of decay generally increases as the ambient temperature is raised above the break-even point (Leverenz 1968). Since the isothermal decay at different temperatures yields information regarding the recombination kinetics (Kathuria and Sunta 1982; Takamori and Dove 1983), it has become a useful tool for studying thermoluminescence peaks of a phosphor (May and Partridge 1964; Sathyamoorthy and Luthra 1978).

We have obtained isothermal decay curves for $\mathrm{MgO}: \mathrm{Li}: \mathrm{Ce} 10^{-2}: \mathrm{Eu} 10^{-3}$ fired in carbon monoxide (Bapat and Sivaraman 1983) at different temperatures. It was noted that the phosphor behaved differently at $480 \mathrm{~K}$. The decay curve showed a usual decrease in intensity up to $15 \mathrm{sec}$. After this the intensity showed a reversal. The rise in intensity continued up to $63 \mathrm{sec}$. Further lapse of time, however, showed a slow decrease in the emission intensity. This feature of the decay viz the rise in intensity to values which should prevail at earlier times, is a surprising result (Bapat and Sivaraman 1985). This result is fairly reproducible within the limits to which the parameters involved i.e. dose of excitation and the temperature can be kept constant.

It is evident that if one takes the phenomenon as a property of the host, one has to account for a large number of electrons in the traps later than the period preceding it. The only source from which electrons could reach the traps emptying at $480 \mathrm{~K}$ would be the traps corresponding to temperatures greater than $500 \mathrm{~K}$. Since the result is reproducible in that the peak occurs at the same time under similar conditions, there would be a regular sequence of events. We therefore hypothesize that: (i) the number of electrons tunnelling into the shallower traps depends on the population of electrons in the deeper traps; (ii) the number of rushing electrons depends on the number of shallower levels available; (iii) electrons from deeper traps rush towards shallower traps 
only if the traps are of the same kind. If these assumptions are valid, it should be possible to affect the position of the peak by changing the extent to which deep traps are occupied (by changing the dose of excitation) and also altering the population of the shallower group using a different ambient temperature.

To verify the first hypothesis, the sample was annealed at $800 \mathrm{~K}$ to empty all the traps up to this temperature. The phosphor was then excited for different times so that the number of deep traps occupied is altered. As envisaged the peak occurs earlier as the excitation dose is increased (figure 1), showing that the tunnelling depends on the number of electrons in deeper traps. The peak intensities were also higher for increased doses of excitation.

If the temperature of decay measurements is raised from 480 to 500 and $520 \mathrm{~K}$ it is expected that the shallower traps would empty at a faster rate and the peak would be expected earlier. Figure 2 shows that the decay pattern confirms this view.

It was observed that there was a threshold $x$-ray dose for observing the peak. If we excite the sample to a lower dose no observable peak is produced. This property helps in identifying the source group of trapped carriers.

Figure 3 shows the thermoluminescence glow runs obtained after a specific dose of excitation at $480 \mathrm{~K}$. The time interval between the cessation of excitation and the start of the glow experiment causes a reduction in the intensity of the TL peak around $540 \mathrm{~K}$ of this sample. The sample was then excited for a time sufficient to populate the deep traps and the TL run was started immediately after switching off the excitation without waiting for any decay. This was to ensure that a reasonable number of shallower traps were still occupied. In the following experiment, after the same exposure at $480 \mathrm{~K}$, the excitation was stopped and the sample was allowed to decay for $1 \mathrm{~min}$ before recording the TL. The TL peak corresponding to $540 \mathrm{~K}$ showed a marked decrease in the latter case indicating that this group had been depopulated. Thus it appears that the TL group at

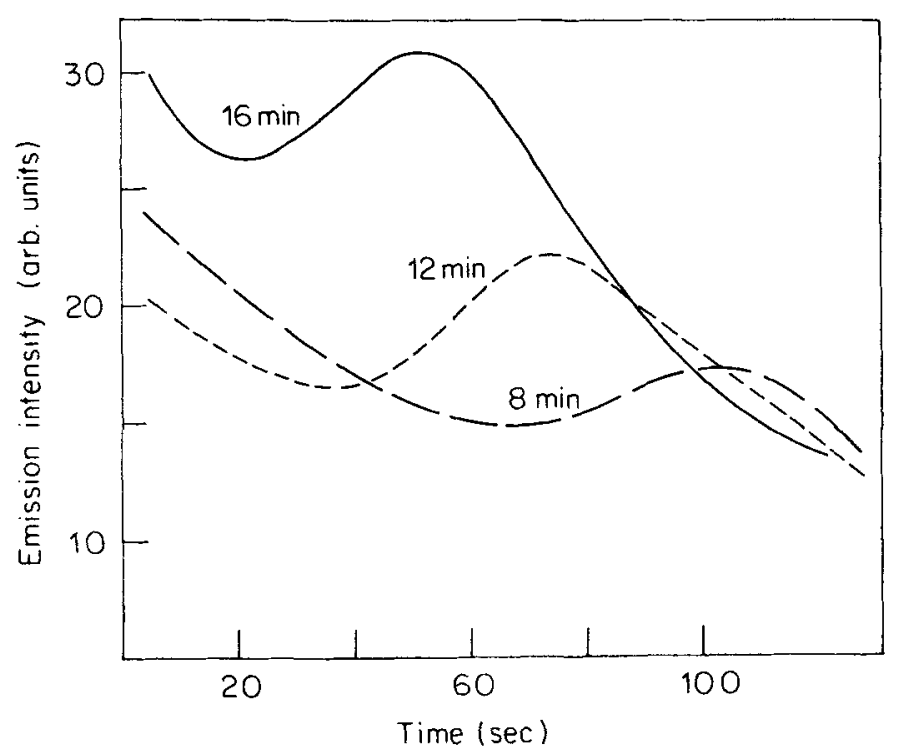

Figure 1. Decay of phosphorescence of $\mathrm{MgO}: \mathrm{Li}: \mathrm{Ce} 10^{-2}: \mathrm{Eu} 10^{-3}$ fired in $\mathrm{CO}$ after different $x$-ray doses. 


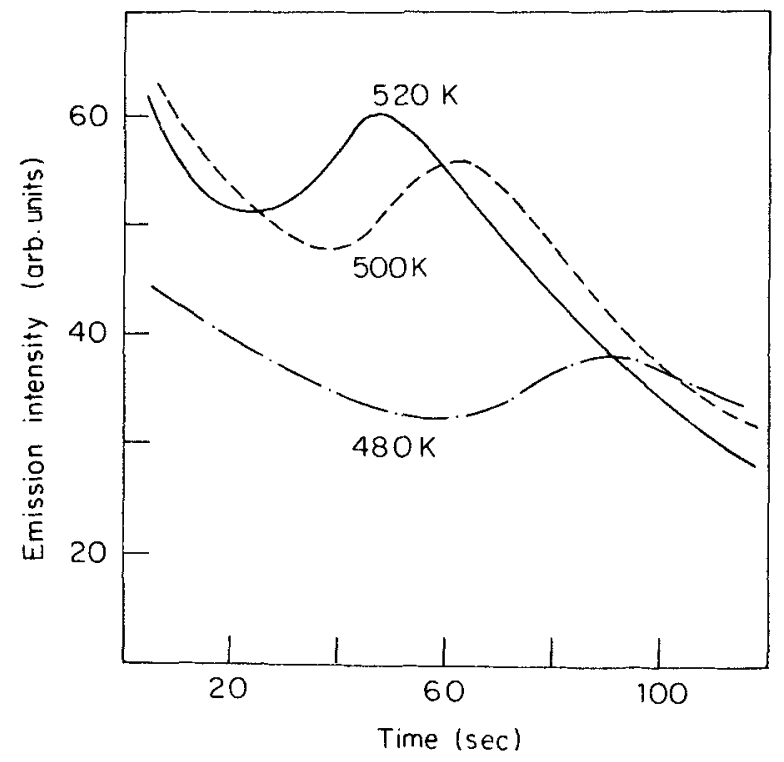

Figure 2. Decay monitored at different ambient temperatures after 10 min dose of $30 \mathrm{kV}$, $10 \mathrm{~mA}$ x-rays.

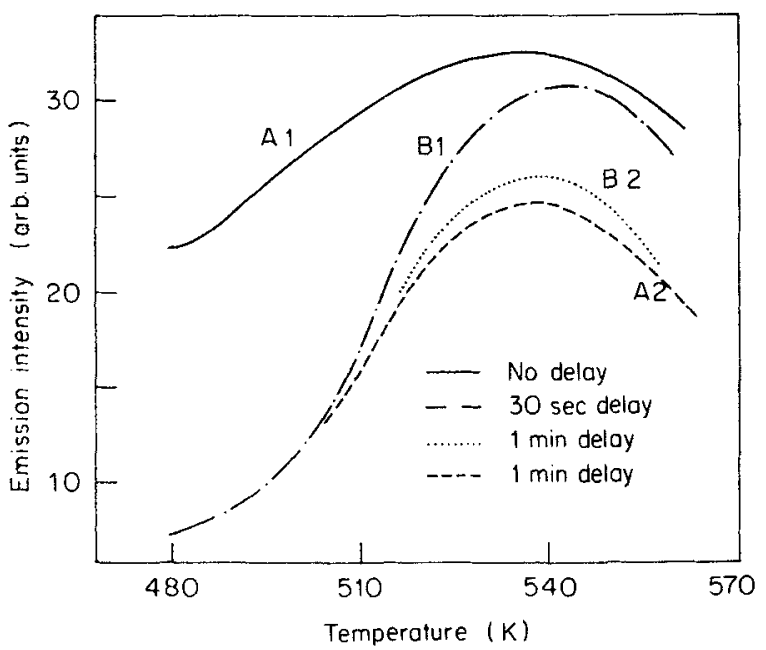

Figure 3. Thermoluminescence glow curves: A1, A2 after saturation excitation; B1, B2 after 3 min excitation.

$540 \mathrm{~K}$ is atleast one of the sources for the electrons being received by the shallower group.

We know from the literature (Kirsh et al 1977) that the trapping group corresponding to $550 \mathrm{~K}$ of $\mathrm{MgO}$ is of electron releasing nature. This being so, if we lower the ambient temperature of decay measurements to a value where hole traps are emptied, 
no such phenomenon should occur. Experiments performed at or below $430 \mathrm{~K}$ give results consistent with the third hypothesis.

This phenomenon was not observed in all our samples. In this connection some general remarks may be in order. One requirement for observing the peak is obviously the condition that at a particular instant the number of carriers tunnelling in must exceed the number being thermally emptied. Another condition would be that apart from the two group of traps being of the same kind, a certain overlap in the wave functions of the carriers at the given temperature should exist. This in turn would depend upon the nature of the two trap groups (potentials) and their actual distribution in the crystal matrix.

Therefore, while this phenomenon is not all too common since certain critical conditions outlined above have to be satisfied, it may not be all that rare. We therefore performed this experiment on a number of systems available to us and found that it occurred in europium-doped $\mathrm{MgO}$ and dyspresium-doped strontium silicate as well. What was more rewarding was that all the experiments conducted on them verified the hypotheses presented earlier.

\section{References}

Bapat M N and Sivaraman S 1983 Indian J. Pure Appl. Phys. 21247

Bapat M N and Sivaraman S 1985 Indian J. Pure Appl. Phys. (in press)

Kirsh Y, Kristianpoller N and Chen Y 1977 Philos. Mag. 35653

Kathuria S P and Sunta C M 1982 J. Phys. D15 497

Leverenz H W 1968 An introduction to luminescence of solids (New York: Dover) p. 256

May C E and Partridge J 1964 J. Chem. Phys. 401401

Sathyamoorthy A and Luthra J M 1978 J. Mater. Sci. 132637

Takamori T and Dove D B $1983 \mathrm{~J}$. Electrochem. Soc. 1301437 